\title{
Cardiotoxicity Assessment After Different Adjuvant Hypofractionated Radiotherapy Concurrently Associated with Trastuzumab in Early Breast Cancer
}

\author{
ELISABETTA BONZANO, MARINA GUENZI and RENZO CORVÒ
}

\author{
Department of Radiation Oncology, IRCCS Policlinico San Martino and University, Genoa, Italy
}

\begin{abstract}
Aim: To evaluate cardiotoxicity in patients with human epidermal growth factor receptor $2+($ HER2 +$)$ breast cancer (29 left-sided, 23 right-sided) treated with adjuvant whole-breast hypofractionated radiotherapy (HRT) concurrently administered with the humanized monoclonal antibody to HER2, trastuzumab. Patients and Methods: From February 2008 to June 2017, 52 patients received three-dimensional conformal RT, with different $H R T$ schemes. Echocardiogram monitoring was used to evaluate the decrease in left ventricular ejection fraction (LVEF). Results: At a median follow-up of 5 years, cardiotoxicity was as follows: among the 15 patients treated with 46 Gy: grade (G) 2 in two (13\%), Gl in three (20\%), and GO in 10 (67\%); in those treated with 39 Gy (16 patients): G1 in five (31\%), and GO in 11 (69\%); among the 21 patients treated with 35 Gy: G2 in one (5\%), G1 in five (24\%), and G0 in 15 (71\%). Conclusion: Trastuzumab was shown to be a safe adjuvant treatment when administered with concomitant HRT since it did not increase cardiotoxicity in those with leftsided breast cancer. No differences in LVEF were observed between the HRT schemes.
\end{abstract}

Nowadays, the standard of care for early breast cancer consists of different adjuvant treatments prescribed after conservative surgery, with the aim of minimizing local recurrence and improving overall survival. In order to achieve this important goal, both systemic and locoregional treatments are essential.

This article is freely accessible online.

Correspondence to: Elisabetta Bonzano, Department of Radiation Oncology, IRCCS Policlinico San Martino and University, Largo R. Benzi 10, 16132 Genoa, Italy. Tel: +39 3381976745, e-mail: elisabettabonzano@gmail.com

Key Words: Hypofractionated radiotherapy (HRT), trastuzumab, breast cancer cardiotoxicity, adjuvant treatment.
Between $15 \%$ and $25 \%$ of breast cancer cases express human epidermal growth factor receptor 2 (HER2), which is related to more aggressive disease, due to the superior capacity of such cells for growth and more rapid cell division. If not treated properly, these patients could be affected by a worse prognosis than those with HER2negative tumors (1).

A better outcome, regarding improvement in survival, has been achieved by prescribing trastuzumab as an adjuvant treatment in patients with HER2-positive breast cancer. Firstly, the critical activity of this humanized monoclonal antibody has been shown in the increase of relapse-free survival time and overall survival in women affected by HER2-positive early breast cancer as reported by major international studies (2-6). In such adjuvant trials, 1-year trastuzumab treatment improved overall survival, directly affecting the risk of recurrence and by extending the time to disease recurrence, when it was prescribed in addition to the standard chemotherapy.

Adjuvant radiotherapy (RT), that is usually delivered concurrently with trastuzumab, has a crucial role in locoregional control of breast cancer after conservative surgery (7).

The concomitant administration of trastuzumab and adjuvant RT is strongly supported by evidence that the greatest benefit from both of these treatments is stronger the sooner they are administered. It is well known that RT can reduce the rate of local recurrence, and trastuzumab can make the difference when administered during early phases of the disease by binding with high affinity to the extracellular domain of HER2 (8). Its efficacy has been proven in the adjuvant setting, such as in patients with metastatic disease $(3,9-11)$. Of great interest, trastuzumab seems to act as a radiosensitizer on breast cancer cells (12). The primary concern of such therapy is the risk of cardiotoxicity due to the combined use of these treatments; asymptomatic reduction in left ventricular ejection fraction (LVEF) is the most frequent form of cardiotoxicity (13). Cardiological follow-up for patients treated with trastuzumab 
consists of LVEF assessment by echocardiography performed before, during and after trastuzumab (14-16). Moreover, each treatment course carries an independent risk of cardiotoxicity $(17,18)$.

The aim of this retrospective study was to identify any additional potential risk due to the concomitant administration of RT and trastuzumab. Although various studies in literature focused on this issue (19-21), our specific purpose was to evaluate acute cardiotoxicity in patients with HER2-positive breast cancer treated with trastuzumab concomitantly with different adjuvant whole-breast hypofractionated radiotherapy (HRT), which is increasingly being adopted worldwide in clinical practice $(22,23)$.

\section{Patients and Methods}

Patients. Fifty-two patients treated with adjuvant HRT for stage I-III (24) breast cancer between February 2008 and June 2017 were retrospectively reviewed. Only patients with less than pT2 and less than pN1a disease were enrolled in this analysis, sub-divided based on the side of breast cancer, the presence of cardiac risk factors at baseline, and the HRT schedule administered. The median age was 64 years.

According to immunohistochemistry data (node-positive, hormone receptor-negative, high Ki67 index and HER2-positive), all patients underwent adjuvant chemotherapy followed by trastuzumab and concomitant HRT.

Cardiac risk factors were carefully analyzed in our study as follows: six patients were smokers, two patients were documented as being affected by hypercholesterolemia, 10 patients suffered from hypertension, and one patient had a diagnosis of atrial fibrillation.

Cardiac toxicity was evaluated by analyzing the LVEF decrease with the following monitoring program: the echocardiogram was assessed at the beginning of the treatment with trastuzumab, every 3 months during trastuzumab treatment, with a 6-monthly followup during the first 5 years and then once a year. LVEF was considered as impaired when below $60 \%$.

Systemic treatments. All patients received adjuvant chemotherapy according to the multidisciplinary breast cancer team decisions. Most commonly used chemotherapy regimens were combinations of epirubicin, cyclophosphamide, 5-fluorouracil or epirubicin plus cyclophosphamide, followed by docetaxel every 3 weeks for three cycles or by weekly paclitaxel for 12 weeks.

Twenty-seven patients with hormone receptor-positive breast cancer underwent hormonal therapy with tamoxifen or aromatase inhibitor due to immunohistochemistry ( $>1 \%$ positive). Trastuzumab was administered every 3 weeks ( $6 \mathrm{mg} / \mathrm{kg}$ after the first cycle of $8 \mathrm{mg} / \mathrm{kg}$ ).

Locoregional treatments. Based on age, patients underwent three different whole-breast adjuvant HRT schemes.

Patients under 40 years old (15 patients) received a total dose of $46 \mathrm{~Gy}$ in 20 fractions, four times a week; women aged between 40 and 46 years (16 patients) received 39 Gy in 13 fractions; and patients older than 46 years (21 patients) underwent 35 Gy administered in 10 fractions, at four fractions a week. These hypofractionated schemes are biologically comparable to the conventional fractionation of 50 Gy in 25 fractions in 5 weeks adopted to control residual microscopic cancer cells after surgery.
All patients received three-dimensional conformal RT to the whole breast in a supine position, and a concomitant weekly boost to the tumor bed was delivered based on their risk factors, such as hormone receptor status, Ki-67 index, margin status, and lymph node status. Overall boost dose depended on different schedules, from minimum $3 \mathrm{~Gy}$ to $7.5 \mathrm{~Gy}$.

Left-sided RT was performed in 29 patients, and right-sided in 23 patients

\section{Results}

All acute cardiotoxicities were assessed according to Common Terminology Criteria for Adverse Events v3 (25). At a median follow-up of 5 years (range=6-120 months), 49 patients $(94 \%)$ remained alive; one patient $(2 \%)$ developed locoregional relapse, and distant metastases occurred in two patients $(4 \%)$.

Our overall results for cardiotoxicity were as follows: among the 15 patients treated with HRT of $46 \mathrm{~Gy} / 20$ fractions: Grade (G) 2 in two (13\%), G1 in three (20\%), and G0 in $10(67 \%)$; in the group of patients treated with HRT of $39 \mathrm{~Gy} / 13$ fractions (16 patients): $\mathrm{G} 1$ in five (31\%), and G0 in 11 (69\%), with no grade 2 cardiotoxicity; among the 21 patients treated with $35 \mathrm{~Gy} / 10$ fractions: $\mathrm{G} 2$ in one $(5 \%)$, G1 in five $(24 \%)$, and G0 in $15(71 \%)$. None of the different HRT schemes used seemed to affect the LVEF rate. Twentyone patients $(40 \%)$ were affected by cardiac risk factors at baseline.

The cardiac tolerance was then evaluated in regard to the breast side irradiated. Left-sided RT was performed in 29 patients $(55 \%)$ : one patient $(3 \%)$ had $\mathrm{G} 2$ cardiotoxicity $(\mathrm{LVFE}=50-40 \%)$, nine patients $(31 \%) \mathrm{G} 1$ (LVFE=60-50\%), and 19 patients $(66 \%)$ G0. Right-sided RT was performed in 23 patients $(45 \%)$ : two patients $(8 \%)$ had $\mathrm{G} 2$, four patients (18\%) G1, and 17 patients (74\%) G0. No G3 reduction of LVEF occurred; $\mathrm{G} 1$ to $\mathrm{G} 2$ rates are similar to literature data.

\section{Discussion}

Major international trials have reported the role of trastuzumab in improving survival and quality of life in women affected by HER2-positive breast cancer, which is associated with a high risk of relapse and death from metastatic disease (2-5). In these studies, trastuzumab was prescribed for 1 year in patients in whom systemic treatments (anthracyclines/taxanes) had already been scheduled; it was started concurrently or sequentially to chemotherapy. The FinHer trial was the only one in which trastuzumab was administered concomitantly with chemotherapy for only 9 weeks. It led to a similar outcome as 1 year of the same regimen (6), but this final result needs further confirmation since it was a very small trial. One year of adjuvant trastuzumab currently remains the standard of care. The use of adjuvant RT delivered with hypofractionation is being 
increased in the adjuvant setting of women with breast cancer submitted to surgery (26). The potential side-effects of combining these new RT schedules with trastuzumab have rarely been investigated and reported (19-21).

The outcomes of our clinical research provide few but interesting findings. Firstly, none of the different HRT schemes used seemed to affect the rate of LVEF decrease. This evidence may be ascribed to our accurate delineation of organs at risk of toxicity in order to spare the greatest heart volume during RT. A safer control of potential radiation-induced risks was achieved thanks to contouring of the heart and left coronary artery, introduced in recent years (27); although this new contouring of organs at risk may reduce cases of cardiac stroke, it probably does not affect LVEF. Finally, toxicities derived from concurrent trastuzumab and RT administration could be considered acceptable, even though a longer follow-up is needed to evaluate the new cardiotoxicity rate after new accurate delineation of organs at risk.

Nowadays, improved treatments have prolonged the lifespan of patients, especially women with breast cancer. Consequently, the survival is often sufficiently long to allow determination of both acute and long-term side-effects induced by adjuvant treatments, including RT and trastuzumab. Cardiotoxicity, amongst others, is a crucial factor to consider and if possible to prevent when a combined therapy is prescribed (27). To our knowledge, by reviewing literature data on this issue, this study is original since it analyzed cardiac toxicity in detail in patients with HER2 positive breast cancer treated with trastuzumab and HRT.

In this experience, on a limited number of patients, trastuzumab was shown to be a safe adjuvant treatment when administered with concomitant HRT, since it did not increase cardiac toxicity, even in the case of left-sided breast cancer. Moreover no differences in LVEF decrease were noted between the three different whole-breast adjuvant HRT schemes.

\section{Conflicts of Interest}

There is no conflict of interest to disclose.

\section{References}

1 Marinko T, Dolenc J and Bilban-Jakopin C: Cardiotoxicity of concomitant radiotherapy and trastuzumab for early breast cancer. Radiol Oncol 48: 105-112, 2014.

2 Piccart-Gebhart MJ, Procter M, Leyland-Jones B, Goldhirsch A, Untch M, Smith I, Gianni L, Baselga J, Bell R, Jackisch C, Cameron D, Dowsett M, Barrios CH, Steger G, Huang C-S, Andersson M, Inbar M, Lichinitser M, Láng I, Nitz U, Iwata H, Thomssen C, Lohrisch C, Suter TM, Rüschoff J, Suto T, Greatorex V, Ward C, Straehle C, McFadden E, Dolci MS, Gelber RD and Herceptin Adjuvant (HERA) Trial Study Team: Trastuzumab after adjuvant chemotherapy in HER2-positive breast cancer. N Engl J Med 353: 1659-1672, 2005.
3 Romond EH, Perez EA, Bryant J, Suman VJ, Geyer CE, Davidson NE, Tan-Chiu E, Martino S, Paik S, Kaufman PA, Swain SM, Pisansky TM, Fehrenbacher L, Kutteh LA, Vogel VG, Visscher DW, Yothers G, Jenkins RB, Brown AM, Dakhil SR, Mamounas EP, Lingle WL, Klein PM, Ingle JN and Wolmark N: Trastuzumab plus adjuvant chemotherapy for operable HER2-positive breast cancer. N Engl J Med 353: 1673-1684, 2005.

4 Slamon D, Eiermann W, Robert N, Pienkowski T, Martin M, Press M, Mackey J, Glaspy J, Chan A, Pawlicki M, Pinter T, Valero V, Liu M-C, Sauter G, von Minckwitz G, Visco F, Bee V, Buyse M, Bendahmane B, Tabah-Fisch I, Lindsay M-A, Riva A, Crown $J$ and Breast Cancer International Research Group: Adjuvant trastuzumab in HER2-positive breast cancer. N Engl J Med 365: 1273-1283, 2011.

5 Spielmann M, Roché H, Delozier T, Canon J-L, Romieu G, Bourgeois H, Extra J-M, Serin D, Kerbrat P, Machiels J-P, Lortholary A, Orfeuvre H, Campone M, Hardy-Bessard A-C, Coudert B, Maerevoet M, Piot G, Kramar A, Martin A-L and Penault-Llorca F: Trastuzumab for patients with axillary-nodepositive breast cancer: results of the FNCLCC-PACS 04 trial. J Clin Oncol Off J Am Soc Clin Oncol 27: 6129-6134, 2009.

6 Joensuu H, Bono P, Kataja V, Alanko T, Kokko R, Asola R, Utriainen T, Turpeenniemi-Hujanen T, Jyrkkiö S, Möykkynen K, Helle L, Ingalsuo S, Pajunen M, Huusko M, Salminen T, Auvinen P, Leinonen H, Leinonen M, Isola J and KellokumpuLehtinen P-L: Fluorouracil, epirubicin, and cyclophosphamide with either docetaxel or vinorelbine, with or without trastuzumab, as adjuvant treatments of breast cancer: final results of the FinHer Trial. J Clin Oncol Off J Am Soc Clin Oncol 27: 5685-5692, 2009.

7 Cao L, Cai G, Xu F, Yang Z-Z, Yu X-L, Ma J-L, Zhang Q, Wu J, Guo X-M and Chen J-Y: Trastuzumab improves locoregional control in HER2-positive breast cancer patients following adjuvant radiotherapy. Medicine 95: e4230, 2016.

8 Swain SM: Adjuvant trastuzumab: Does time really matter? Oncologist 18: 490-492, 2013.

9 Slamon DJ, Leyland-Jones B, Shak S, Fuchs H, Paton V, Bajamonde A, Fleming T, Eiermann W, Wolter J, Pegram M, Baselga $\mathrm{J}$ and Norton L: Use of chemotherapy plus a monoclonal antibody against HER 2 for metastatic breast cancer that overexpresses HER2. N Engl J Med 344: 783-792, 2001.

10 Watanabe J, Ito Y, Saeki T, Masuda N, Takano T, Takao S, Nakagami K, Tsugawa K, Nakagawa S, Kanatani $K$ and Nakayama T: Safety evaluation of trastuzumab emtansine in Japanese patients with HER2-positive advanced breast cancer. In Vivo 31: 493-500, 2017.

11 Fujii T, Horiguchi J, Yanagita Y, Koibuchi Y, Ikeda F, Uchida N, Kimura M and Gunma Breast Clinical Conference Study Group (GBCCSG): Phase II study of S-1 plus trastuzumab for HER2positive metastatic breast cancer (GBCCSG-01). Anticancer Res 38: 905-909, 2018.

12 Liang K, Lu Y, Jin W, Ang KK, Milas L and Fan Z: Sensitization of breast cancer cells to radiation by trastuzumab. Mol Cancer Ther 2: 1113-1120, 2003

13 Jones AL, Barlow M, Barrett-Lee PJ, Canney PA, Gilmour IM, Robb SD, Plummer CJ, Wardley AM and Verrill MW: Management of cardiac health in trastuzumab-treated patients with breast cancer: Updated United Kingdom National Cancer Research Institute recommendations for monitoring. Br J Cancer 100: 684-692, 2009. 
14 Johri AM, Picard MH, Newell J, Marshall JE, King MEE and Hung J: Can a teaching intervention reduce interobserver variability in LVEF assessment? A quality control exercise in the echocardiography lab. JACC Cardiovasc Imaging 4: 821-829, 2011.

15 Lang RM, Bierig M, Devereux RB, Flachskampf FA, Foster E, Pellikka PA, Picard MH, Roman MJ, Seward J, Shanewise JS, Solomon SD, Spencer KT, Sutton MSJ, Stewart WJ, Chamber Quantification Writing Group, American Society of Echocardiography's Guidelines and Standards Committee and European Association of Echocardiography: Recommendations for chamber quantification: a report from the American Society of Echocardiography's Guidelines and Standards Committee and the Chamber Quantification Writing Group, developed in conjunction with the European Association of Echocardiography, a branch of the European Society of Cardiology. J Am Soc Echocardiogr Off Publ Am Soc Echocardiogr 18: 1440-1463, 2005.

16 Davis CC, Zelnak A, Eley JW, Goldstein DA, Switchenko JM and McKibbin T: Clinical utility of routine cardiac monitoring in breast cancer patients receiving trastuzumab. Ann Pharmacother 50: 712-717, 2016.

17 Hooning MJ, Botma A, Aleman BMP, Baaijens MHA, Bartelink H, Klijn JGM, Taylor CW and van Leeuwen FE: Long-term risk of cardiovascular disease in 10-year survivors of breast cancer. J Natl Cancer Inst 99: 365-375, 2007.

$18 \mathrm{Wu}$ Y-Y, Huang T-C, Tsai T-N, Chen J-H, Dai M-S, Chang P-Y, Ho C-L, Ye R-H, Chung T-R, Chen Y-C and Chao T-Y: The clinical efficacy and cardiotoxicity of fixed-dose monthly trastuzumab in HER2-positive breast cancer: a single institutional analysis. PloS One 11: e0151112, 2016.

19 Cao L, Cai G, Chang C, Yang Z-Z, Feng Y, Yu X-L, Ma J-L, Wu J, Guo X-M and Chen J-Y: Early cardiac toxicity following adjuvant radiotherapy of left-sided breast cancer with or without concurrent trastuzumab. Oncotarget 7: 1042-1054, 2015.

20 Meattini I, Cecchini S, Muntoni C, Scotti V, De Luca Cardillo C, Mangoni M, Bonomo P, Nori J, Casella D, Simoncini R, Orzalesi L, Bianchi S and Livi L: Cutaneous and cardiac toxicity of concurrent trastuzumab and adjuvant breast radiotherapy: a single institution series. Med Oncol 31: 891, 2014.
21 Belkacemi Y, Gligorov J, Ozsahin M, Marsiglia H, De Lafontan B, Laharie-Mineur H, Aimard L, Antoine E-C, Cutuli B, Namer $M$ and Azria D: Concurrent trastuzumab with adjuvant radiotherapy in HER2-positive breast cancer patients: acute toxicity analyses from the French multicentric study. Ann Oncol 19: 1110-1116, 2008.

22 Corvò R, Ricchetti F, Doino D, Torielli P, Agostinelli S, Cavagnetto F, Giannelli F, D'Alonzo A, Vagge S, Belgioia L and Guenzi M: Adjuvant hypofractionated radiotherapy with weekly concomitant boost for women with early breast cancer: the clinical experience at Genoa University. Anticancer Res 30: 4749-4753, 2010.

23 Guenzi M, Vagge S, Azinwi NC, D’Alonzo A, Belgioia L, Garelli S, Gusinu M and Corvò R: A biologically competitive 21 days hypofractionation scheme with weekly concomitant boost in breast cancer radiotherapy feasibility acute sub-acute and short term late effects. Radiat Oncol 5: 111, 2010.

24 Edge SB and Compton CC: The American Joint Committee on Cancer: the 7th edition of the AJCC cancer staging manual and the future of TNM. Ann Surg Oncol 17: 1471-1474, 2010.

25 Trotti A, Colevas AD, Setser A, Rusch V, Jaques D, Budach V, Langer C, Murphy B, Cumberlin R, Coleman CN and Rubin P: CTCAE v3.0: development of a comprehensive grading system for the adverse effects of cancer treatment. Semin Radiat Oncol 13: 176-181, 2003.

26 Hou H-L, Song Y-C, Li R-Y, Zhu L, Zhao L-J, Yuan Z-Y, You J-Q, Chen Z-J and Wang P: Similar outcomes of standard radiotherapy and hypofractionated radiotherapy following breastconserving surgery. Med Sci Monit Int Med J Exp Clin Res 21: 2251-2256, 2015.

27 Feng M, Moran JM, Koelling T, Chughtai A, Chan JL, Freedman L, Hayman JA, Jagsi R, Jolly S, Larouere J, Soriano J, Marsh R and Pierce LJ: Development and validation of a heart atlas to study cardiac exposure to radiation following treatment for breast cancer. Int J Radiat Oncol Biol Phys 79: 10-18, 2011.

Received March 15, 2018

Revised April 14, 2018

Accepted April 17, 2018 